\title{
ENHANCEMENT TECHNIQUES FOR DATA WAREHOUSE STAGING AREA
}

\author{
Mahmoud El-Wessimy, Hoda M.O. Mokhtar, Osman Hegazy \\ Faculty of Computers and Information, Cairo University, Cairo-Egypt
}

\begin{abstract}
Poor performance can turn a successful data warehousing project into a failure. Consequently, several attempts have been made by various researchers to deal with the problem of scheduling the ExtractTransform-Load (ETL) process. In this paper we therefore present several approaches in the context of enhancing the data warehousing Extract, Transform and loading stages. We focus on enhancing the performance of extract and transform phases by proposing two algorithms that reduce the time needed in each phase through employing the hidden semantic information in the data. Using the semantic information, a large volume of useless data can be pruned in early design stage. We also focus on the problem of scheduling the execution of the ETL activities, with the goal of minimizing ETL execution time. We explore and invest in this area by choosing three scheduling techniques for ETL. Finally, we experimentally show their behavior in terms of execution time in the sales domain to understand the impact of implementing any of them and choosing the one leading to maximum performance enhancement.
\end{abstract}

\section{KEYWORDS}

DataWarehouse, ETL,Data Loadingschedules, ETL optimization

\section{INTRODUCTION}

Lately data warehousing (DW) has gained a lot of attention both from both the industry and research communitycommunities. From the industrial perspective, building an information system for the huge data volumes in any industry requires lots of resources as time and money. Unless those resources add to the industry value, such systems are worthless. Thus, people require that information systems should be capable to provide extremely fast responses to different queries specially those queries that affect decision making. From the research perspective, researchers find that due to the increasing need and value of for efficient data warehouses, it is still a fruitful research direction where further improvements can be added., further investigation in data warehouses performance and technqiues are still needed and present fruitful research directions.

In [1], the authors show how data warehousing systems address the issue of enabling managers to acquire and integrate information from different sources, and to efficiently query very large

DOI : 10.5121/ijdkp.2013.3601 
databases. The authors mention that during challenging times, good decision-making becomes critical. The best decisions are made when all the relevant data is taken into consideration.Today, the biggest challenge in any organization is to achieve better performance with least cost, and to make better decisions than competitors. That is why data warehouses are widely used within the largest and most complex businesses in the world. According to the authors in [2], a data warhouse (DW) is a collection of consistent, subject-oriented, integrated, time-variant, nonvolatile data along with processes on them, which are based on current and historical information that enable people to make decisions and predictions about the future. The DW is suitable for direct querying and analysis, and it stands as a source for building logical data marts oriented to specific areas of an enterprize. Due to the importance of enhancing the data quality several vendors presented a number of quality measurement tools such as IBM InfoSphere Information Analyzer, Oracle Data Profiling, open source as DataCleaner, and Microsoft Data Profiler, and others.

In this paper, Tto measure the quality of our results we selected Microsoft data profiler tool due to its simplicity and richness of the analysis provided [3,4]. Generally, a data profile is a collection of aggregate statistics about the data in the different relations (tables) that might include: number of rows in a table, and/or Count count of distinct values in a column, number of "nullNULL" or missing values in a column, the distribution of values along a column, as welll as the strength of the functional dependency of one column on another (this will help in choosing the Primary primary Keykey) on another.The statistics that a data profile provides gives the information that one needs in order to effectively minimize the quality issues that might occur from using heterogeneous source data. Inspired by the importance of building efficient data warehouses, in this work we investigate the use of domain semantics to enhance the extraction and transformation phases in the staging area. We focus on the "Sales" bussiness area domain due to its simplicity and familiarity. In Addtion, loading is the process of populating the data into the data warehouse DW. As simple as this process may seem to be, it can't be replaced, whether we are dealing with flat files, excel sheets, or relational databases, it all comes to delivering the data to its final repository to have one single version of truth enabling to make the right decision at the right time. Thus, enhancing the loading process and a crucial ingrdient in the overall DW enhancement process.

Authors in [5] explored the scheduling phase from system memory utilization perspective as they expressed in their paper that since having many applications involving continuous data streams, data arrival is burst and data rate fluctuates over time. Systems that seek to give rapid or real-time query responses in such an environment must be prepared to deal gracefully with bursts in data arrival without compromising system performance. So their goal was to utilize resources during times of peak load by choosing the appropriate scheduling strategy which can have significant impact on the run time system memory usage as well as output latency. Besides, many researches were interested in the relationship between data-to-application flow and transferring data to traditional data warehouse while managing system resources. Others investigated the possibility to enhance the load process by not only focusing on the best scheduling technique but also analyzing and understanding the behavior and structure of its repository to deliver the data in the most efficient manner.

In this direction, authors in [6] mentioned that a simple low-cost shared-nothing architecture with horizontally fully-partitioned facts can be used to speedup response time of the data warehouse significantly and they concluded after experiments that, although it is not possible to guarantee linear speedup for all query patterns, workload-friendly placement can prevent very low speedup 
and provide near to linear speedup for most queries in Node Partitioned Data Warehouses. Our goal is to continue the effort towards an enhanced data warehousing performance through its final phase "loading". We are motivated by the fact that in real life important information that is delivered late results in making inaccurate decisions. In this context, we explore three scheduling techniques (First-In-First-Out (FIFO), Minimum Cost, and Round Robin (RR) based on time and records) for scheduling the ETL process. We experimentally show their behavior in terms of execution time with our sales data and discuss the impact of their implementation.

The rest of the paper is arranged as follows: Section 2 presents a literature overview of related work. Section 3 introduces the problem discussed in this paper.Section 4 presents our semanticsbased extraction algorithm for the sales bussiness area. Section 5 discusses the semantics-based transformation algorithm .Section 6 introduces our proposed data loading scheduling algorithms. Section 7 presents our scheduling experimental results. Finally, section 8 concludes and presents possible directions for future work.

\section{RELATED WORK}

Today, DW and on-line analytical processing (OLAP) are essential elements for decision support. In [7], the authors delved into the logical optimization of the ETL processes, modeling it as a statespace search problem. They considered each ETL workflow as a state and presented the state space through a set of correct state transitions. Moreover, some algorithms were provided towards the minimization of the execution cost of the ETL workflow. In addition, there has been a lot of work to optimize the performance of relational data warehouses. Authors in [8] suggested combining three major techniques that can be used for this objective: enhanced index schemes (join indexes, bitmap indexes), materialized views, and data partitioning. Currently, the existing research prototypes or products use materialized views alone or indexes alone or combination of them, but none of the prototypes use all three techniques together. In the paper the authors showed by a systematic experimental evaluation that the combination of these three techniques reduces the query processing cost and the maintenance overhead significantly.

On the other hand, authors in [9] focused on proposing an ontology-based ETL framework for covering schema integration as well as semantic integration. In their approach, beside the schemabased semantics in Common Warehouse Metamodel (CWM), they claim that semantic interoperability in ETL processes can be improved by means of an ontology-based foundation for better representation, and management of the underlying domain semantics. Several other work considered the ontology-based ETL including $[10,11]$. The last phase in the ETL stage after extracting and transforming data is the load phase. The objective of the load phase is simple "load the data into the end target" which is usually the data warehouse (DW). The rate of exporting data may vary from daily, weekly or monthly basis. Basically, the main challenge resides in delivering the right data at the right time. In this direction many researches were conducted to optimize the transfer process. Authors in [12] investigated the best loading method by presenting a methodology for achieving useful-time data warehousing by enabling continuous data integration, while minimizing impact of query execution on the user end of the DW. This is achieved by data structure replication and adapting query instructions in order to take advantage of the new schemas, while executing the best previously determined continuous data integration methods.

On the other hand, authors in [13] realized that with the increase in the updates, previously fast queries tend to slow down considerably. However, depending on the user requirements, the response 
time or the data quality can be improved by scheduling the queries and updates appropriately and thus they mentioned that if both criteria are to be considered simultaneously, we are faced with a so-called multi-objective optimization problem. Hence, they developed a scheduling approach that provides the optimal schedule with regard to the user requirements at any given point in time supported by their evaluation for the scheduling in an extensive experimental study. In addition, authors in [14] presented an approach for automating the derivation of incremental load jobs based on equational reasoning. They started by reviewing existing Change Data Capture techniques as well as loading facilities for data warehouse refreshment then based on them they provided transformation rules for the derivation of incremental load jobs. In the same context of data loading, authors in [15] considered the issue of bulk loading large data sets for the UB-Tree, a multidimensional clustering index which inherits all good properties of B-Tree. Specially in data warehousing, data mining and OLAP it is necessary to have efficient bulk loading techniques, because loading doesn't occur continuously, but only from time to time with usually large data sets. The authors thus proposed two techniques, one for initial loading, which creates a new UBTree, and one for incremental loading, which adds data to an existing UB-Tree. Both techniques try to optimize both the I/O and CPU cost.

Authors in [16] presented a GUI based ETL procedure for the continuous loading of the data in the active data warehouse. The main idea is to enable continuous data integration along with minimizing the impact on query execution at the user end. Despite the researches published and how each try to contribute in the enhancement of the loading process in the DW, we must not ignore the fact that we are not dealing with just one type of data, so authors in [17] proposed a new technique called selective load to handle big dimensions in distributed data warehouses by maintaining nearly linear speed up in query execution time. Finally, authors in [18] dealt with the problem of scheduling off-line ETL scenarios, aiming at improving both the execution time and memory consumption without allowing data losses. In doing so, they assessed a set of scheduling policies for the execution of ETL work flows like minimum cost and other techniques and analyzed their behavior with different input sizes.

\section{PROBLEM DEFINITION}

Sales and marketing are considered slippery territory as they deal with people (customers), and people often do allow their emotions to drive their decision-making process. However, this characteristic does not hinder the ability to rationally define measure, analyze, and improve this important business domain. Nevertheless, it is true that most organizations' failure is usually due to the lack of confidence in the information gathered about production/people, along with failure to acquire the right data at the right time. Hence, in order to overcome this situation, an organization should define the major factors affecting its sales and profit such as lack of proper strategy, consuming the time of salespeople with unnecessary tasks, and defining the right products that will return high ROI. Consequently, in this work we are interested in finding a way to assist the sales industry enhancing their repository that basically contains all necessary data about their productivity. However, due to the fact that there is a wide spectrum of possible research directions including: enhancing the performance of the staging area, enhancing data analysis, focusing on design issues, and studying Business Intelligence (BI) along with many others, we decided to start exploring the extraction and transformation processes of raw data acquired from the operational level from heterogeneous data sources and how it could be improved to finally load high quality data into the DW as well as the best approach to load it. In addition, in order to capture the added 
International Journal of Data Mining \& Knowledge Management Process (IJDKP) Vol.3, No.6, November 2013

value and to benefit from enhancing the data warehouse staging area, several contributing dimensions need to be considered:

1) Human Factor: Prior knowledge about the type of data is very important. Given the fact that there are a lot of changes going on the percentage of understanding of the raw operational data to be extracted is one of the factors impacting in the quality of the extraction process.

2) General Ontologies: Those Ontologies represent general rules and guidelines for the ETL phases. Those rules are usually based on the business domain considered. Hence, we gathered the most commonly used extraction ontologies, refined, and tailored them to our problem domain (i.e. the sales domain) to eliminate non-relevant data and achieve higher quality of extracted data.

3) Results from Statistics and Quality Measures: Realizing that every process costs time, and in order to improve the human factor; we used a data quality tool to provide us with a better understanding of the data in hand in terms of the inner relationship within data per table and outer relationship among different tables. Those tools generally enable us to evaluate the distribution of data and determine if there exists any pattern in the data. Such characteristics in the data help us to determine if there any candidate primary keys that require us to apply architecture design change, or to change the size of data.

In general, the advantage of the tool used is cumulative as in the transformation stage the results reflect an improved version of data quality after extraction. Integrating those tools and dimensions we managed in this work to propose an algorithm to efficiently perform each of the extract and transform phases

Example 1: Consider a Sales DW with the star schema shown in Figure 1. The schema has a central Sales fact table along with 7 dimension tables. The relationships among the schema's tables are also shown in the figure. Using a data profiling tool, the current behavior of the "Address" table is shown in Table 1.It is clear that although there are several candidate keys for the Address table, only one attribute has $100 \%$ key strength. This observation resulted in one main functional dependency that hinders future changes in the table structure. In addition, AddressLine2 field contains a high percentage of "NULL" values, whereas we can see that there are a huge number of distinct values for our candidate key column AddressLine1. 
International Journal of Data Mining \& Knowledge Management Process (IJDKP) Vol.3, No.6, November 2013

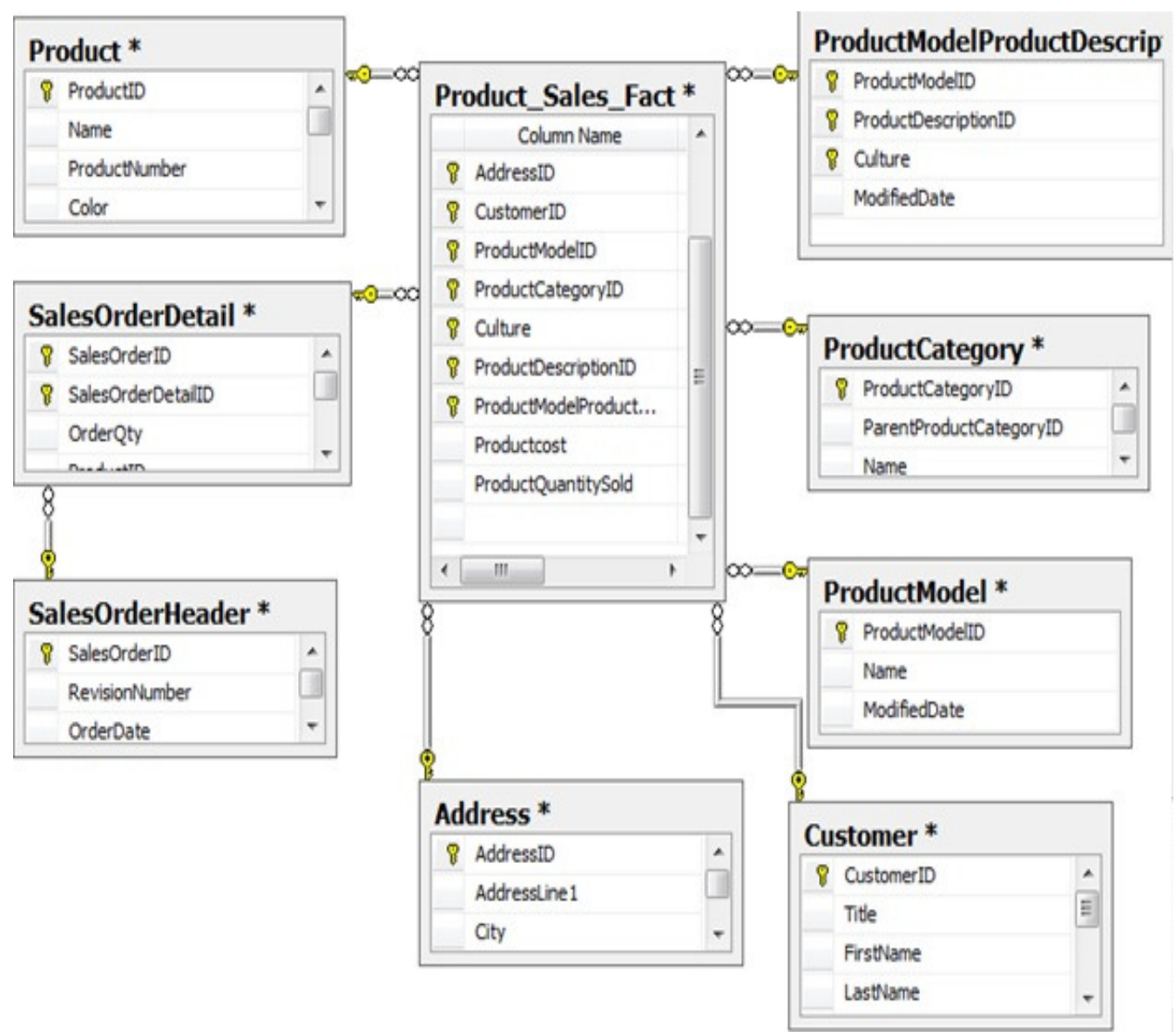

Figure 1. A Simplified Sales Star Schema

\begin{tabular}{|c|c|c|}
\hline Address Table & \multicolumn{2}{|l|}{ Before Extraction } \\
\hline \multirow{4}{*}{$\begin{array}{l}\text { 1- } \begin{array}{l}\text { Number of } \\
\text { Candidate Keys } \\
\text { and Confiderce }\end{array}\end{array}$} & Key Colımns & Key Strergth \\
\hline & Addrassid. & $100.0 \mathrm{co0} \%$ \\
\hline & AddressLine1 & $99.99 \in 7 \%$ \\
\hline & Postal Code & $99.9023 \%$ \\
\hline 2- Column Null Ratio & \multicolumn{2}{|c|}{ AddressLine2 $>99.9927 \%$} \\
\hline \multirow{4}{*}{$\begin{array}{l}\text { 3- Top Column Value } \\
\text { Distribution }\end{array}$} & Column & Number of Distirct Values \\
\hline & Adcress: & 150450 \\
\hline & Adcress_ire 1 & 150445 \\
\hline & PostalCode & 150303 \\
\hline $\begin{array}{l}\text { 4- Functional } \\
\text { Dependency }\end{array}$ & \multicolumn{2}{|c|}{$\begin{array}{l}\text { Cnly the Add ressl2 have full functional dependency } 100 \% \text { whereas } \\
\text { Adcress_ire } 1 \text { and Postal code were below it slightly }\end{array}$} \\
\hline
\end{tabular}

Table 1. Behavior of Address Table

\section{DATA EXTRACTION PAHSE}

The purpose of the extract phase is to start with operational tables (relations) from heterogeneous data sources and then using the pre-defined ontologies produce filtered and customized extracted 
tables that fit with user vision towards the pursuit of enhanced DW. Our proposed algorithm for this phase of the staging area takes as input all operational data denoted by $\mathrm{T}_{\mathrm{op}}$. As well as the type of desired relationships between tables to include denoted by $\mathrm{T}_{\mathrm{R}}$, list of table's names denoted by $\mathrm{T}_{\mathrm{N}}$, along with their assigned relative weights denoted by $\mathrm{T}_{\mathrm{W}}$. The relative weights reflect the table's priority according to the user decision. Finally, we also take as input lists of attributes "A" to extract per table " $\mathrm{T}$ " denoted by $\mathrm{T}_{\mathrm{A}}$. In brief, the algorithm starts by considering each table $\mathrm{T}_{\mathrm{S}}$ in a descending order according to the relative weight $\mathrm{T}_{\mathrm{SW}}$ (weights are assigned using a priority rule), and checks if the table's relationship type $\mathrm{T}_{\mathrm{SR}}$ belongs to the table relationship pre-defined types $T_{S R} \square T_{R}$ (part of or distinct). Then, the same procedure is run but on the attribute level where each current attribute $A_{C}$ is checked if it belongs to the list of attributes to be extracted $A_{C} \square$ $\mathrm{T}_{\mathrm{A}}$. Next, for each table, different rules and criteria are generated for the values per column. Those rules and criteria are designed based on the semantics of the data and are the main reason behind achieving high quality extracted data at the end. To determine the extraction priority of each table there are a number of possible approaches:

1) Based on tables size measured by taking the average length of all columns (using data profile statistics), then assigning prioritized weights (i.e. the order to be executed in).

2) Based on descending order of the percentage of occurrence of null values.

3) Based on the percentage of functional dependencies.

However in our approach we basically apply another measurement based on the number of relationships the examined table is involved in. This criterion makes us begin with the Sales Order Header then Sales Order Detail, etc. Our proposed algorithm is shown below.

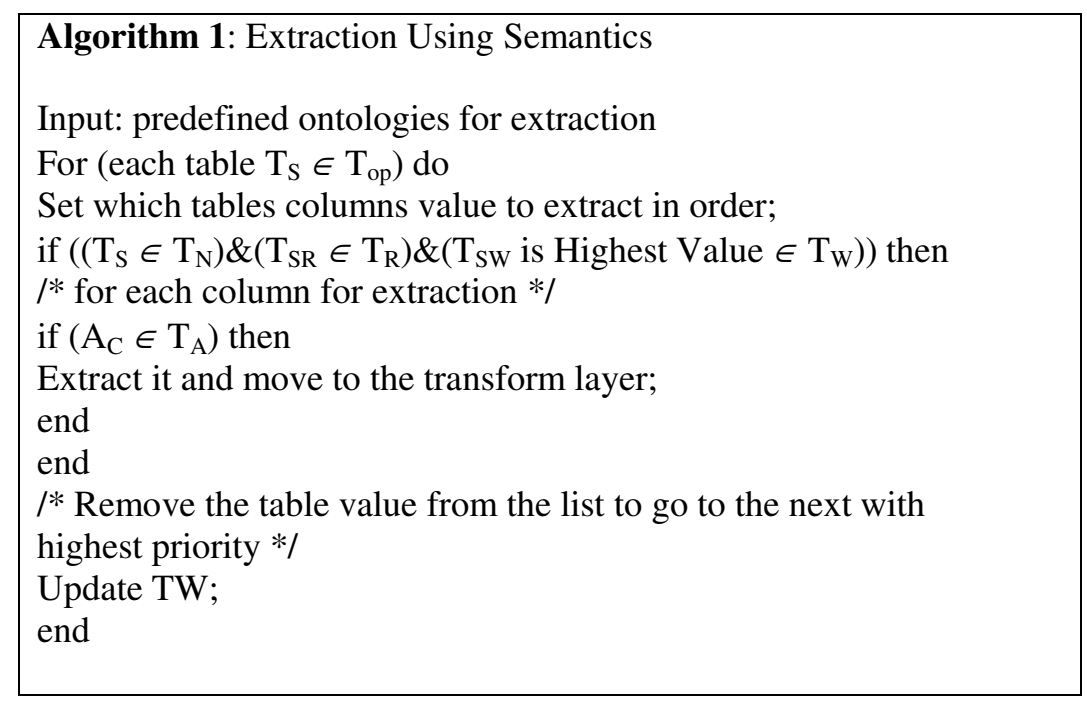

Example 2. The presented extract algorithm is then applied on all tables. Using our previous "Sales" schema, we apply the algorithm on the Address table introduced in Example 1. The initial "Address" table data profile statistics is shown in Table 1. In this example we show how the general semantics-based extraction algorithm is tailored for extracting the "Address" table in our Sales schema. 


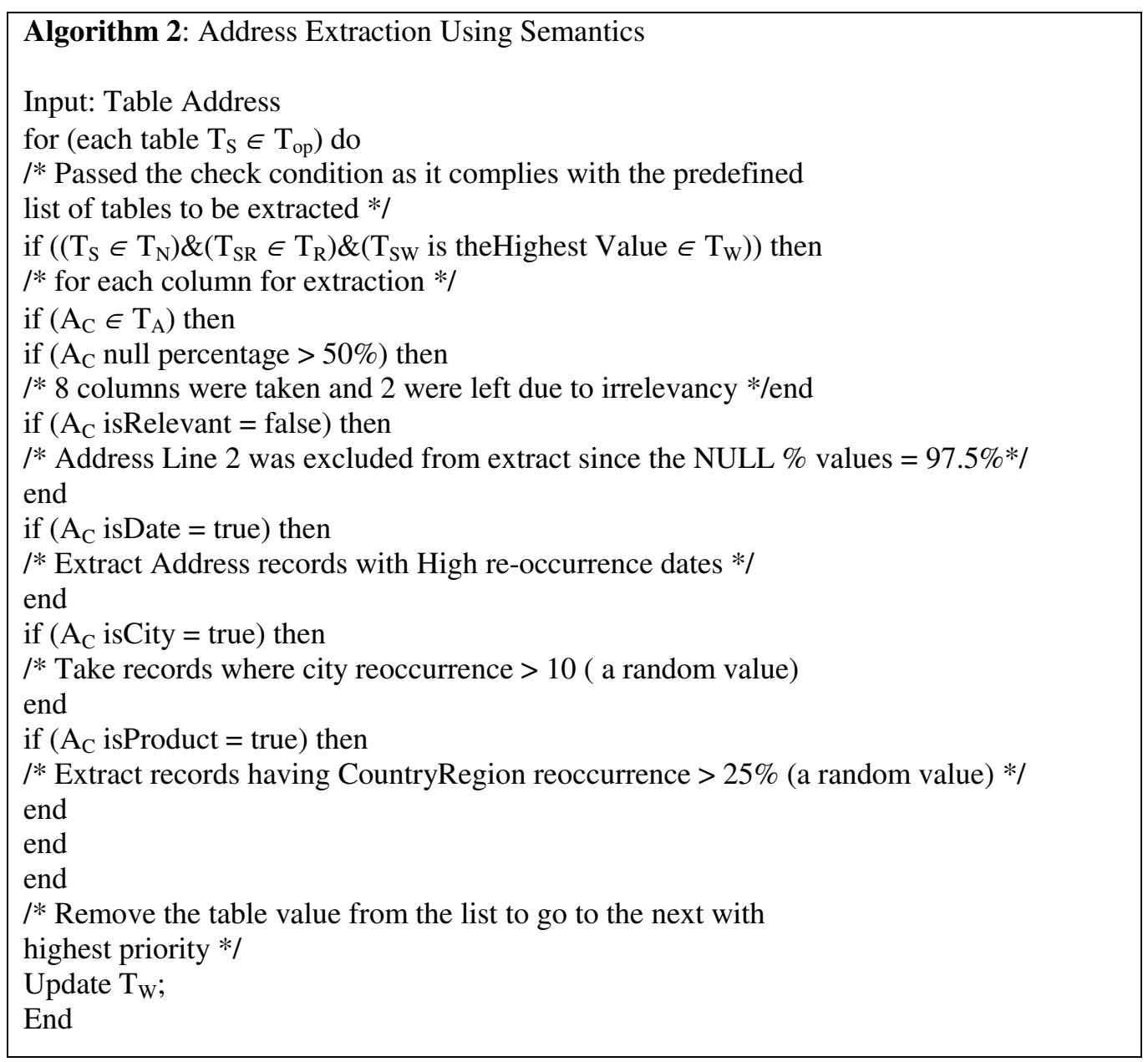

We present a more detailed view of the "Address" table in Figure 2.

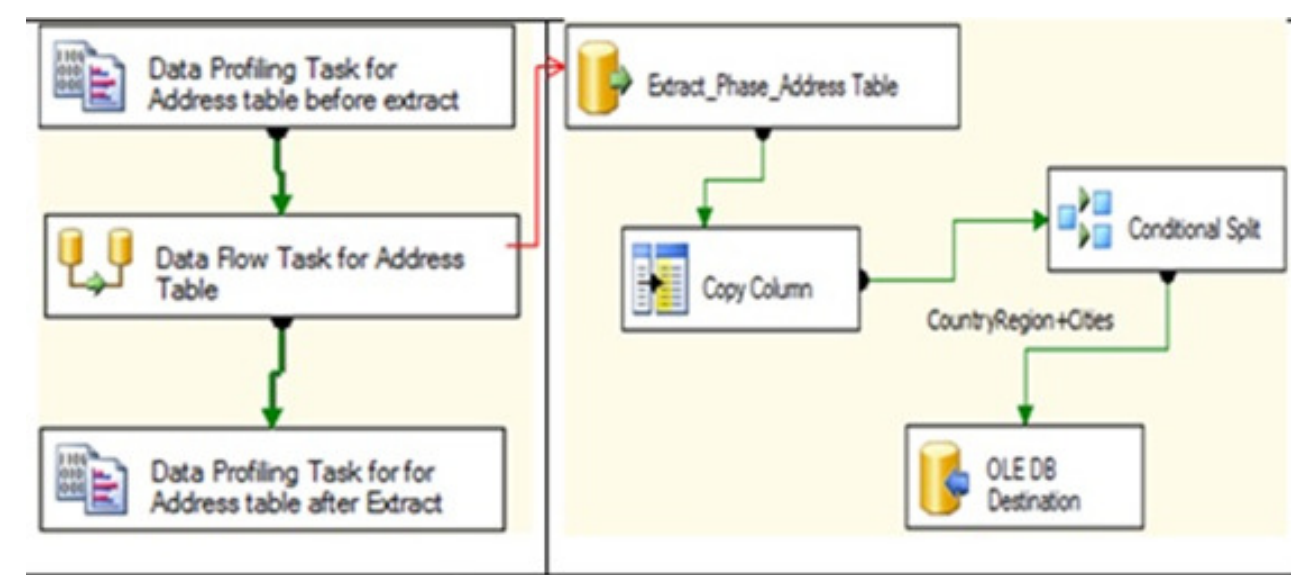

Figure 2. Deeper view for Address Table Extraction 
After executing the semantics-based extraction algorithm we obtain a new data profile statistics for the "Address" table as shown in Table 2.

\begin{tabular}{|c|c|c|}
\hline Address Table & \multicolumn{2}{|l|}{ After Extraction } \\
\hline \multirow{4}{*}{$\begin{array}{l}\text { 1- Number of Candidate Keys and } \\
\text { Confidence }\end{array}$} & Key Columns & Key Strength \\
\hline & Addressin & $100 \%$ \\
\hline & AddressLine1 & $100 \%$ \\
\hline & Postalcode & $99.9740 \%$ \\
\hline 2- Column Null Ration & \\
\hline \multirow[t]{4}{*}{ 3- Top Column Value Distribution } & Column & Number of Distinct Values \\
\hline & Addressid & 150062 \\
\hline & AddressLine1 & 150062 \\
\hline & RostalCode & 150023 \\
\hline 4- Functional Dependency & \multicolumn{2}{|c|}{$\begin{array}{l}\text { Now there are } 2 \text { columns instead of } 1 \text { having } \\
100 \% \text { columns depending on them which are } \\
\text { AddressiD and AddressLine } 1 \text { beside } \\
\text { improvement in Postalcode. }\end{array}$} \\
\hline
\end{tabular}

Table 2. Behavior of Address Table after Extraction

If we compare the number of candidate keys before (Table 1) and after (Table 2), we will notice that they have been improved since AddressLine1 field became as strong as the primary key AddressID with $100 \%$ key strength. This observation also resulted in two main functional dependencies instead of one and this was due to several factors such as the elimination of AddressLine2, whereas we can still see that there are a huge number of distinct values for our candidate keys.

\section{DAta Transformation PAhSE}

Transforming data is the second step in the ETL chain after extracting the operational data. Our proposed algorithm for this phase takes as input the enhanced data from "extraction" phase, an updated list of table's weight for the transformation phase $\mathrm{T}_{\mathrm{rw}}$, and a set of predefined ontologies (restrictions/rules) P for each field values to finally obtain tables that are ready for loading to its final repository. The proposed algorithm is continuously recurring by starting with the highest weight table in Trw, then stepping into 2 inner stages: cleansing and conforming. In the cleansing step, there is a check for the null values in each column, if any null values are found, certain adjustments will be applied; otherwise the conformation stage begins. In the conformation stage, the current attribute $A_{C}$ value $A_{C} . V$ is initially assessed whether it matches with $P$ or not. Then, another assessment is performed to check if the attribute length violates the predefined length range, finally the attribute's value $\mathrm{V}$ is checked for validity. In addition, a final post condition check is applied to evaluate table row count reasonability (e.g. if the number of records is more than expected, which probably means something went wrong like Cartesian product rather than join). In addition, in order to monitor the data integrity an error log table is created to track table's records that did not match the predefined rules and conditions $\mathrm{P}$ while including an informative warning for database administrator to take proper action towards it to rectify. Our proposed algorithm is shown below. 


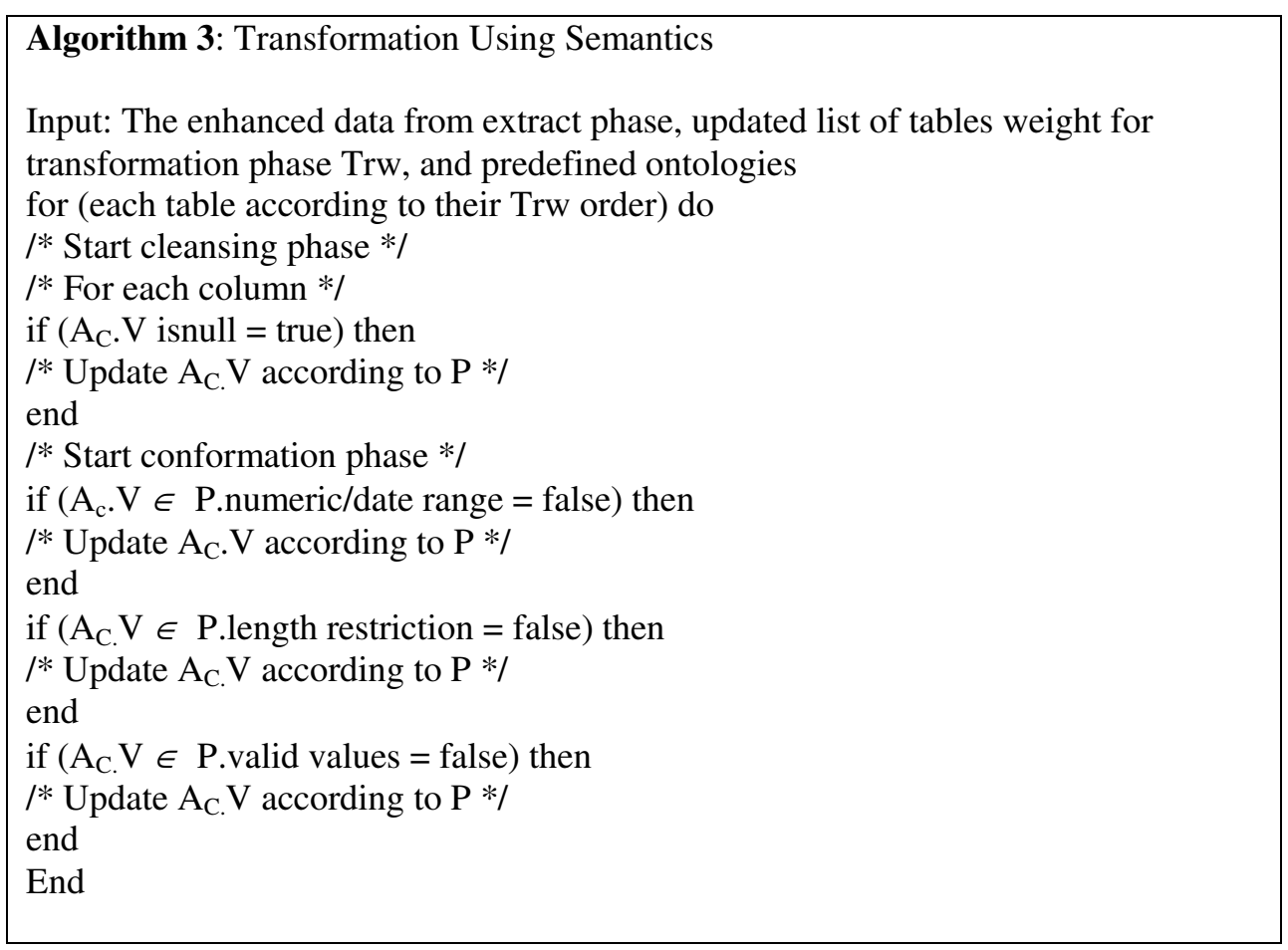

Example 3. Continuing with our "Sales" schema. This example reflects further enhancements in the "Address" table after the transformation phase. The example starts by introducing the below adjusted algorithm, as well as Figure 3 which demonstrates a deeper view of the "Address" table. We then present an analysis of the impact of applying the proposed algorithm on the transformation phase performance.

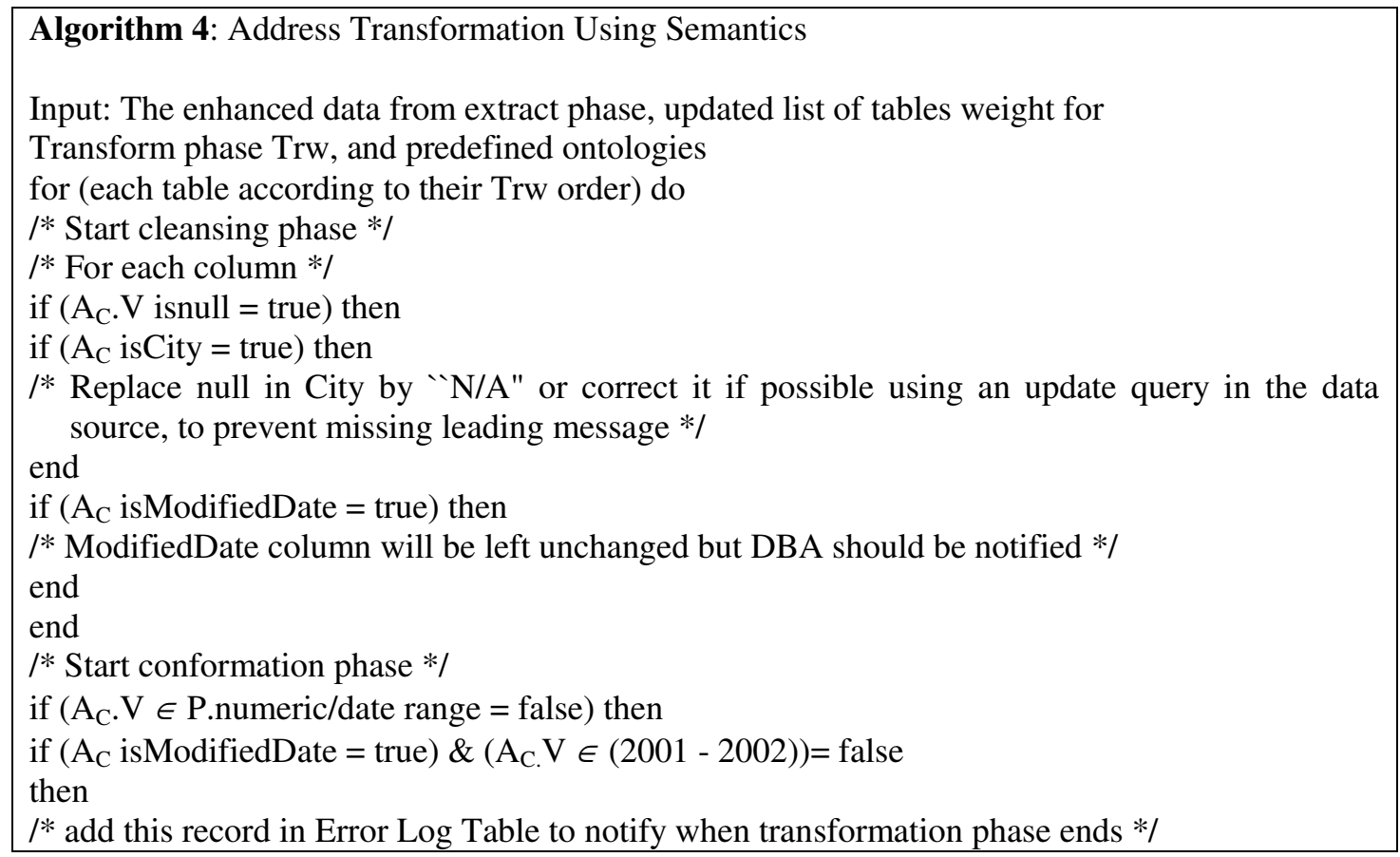



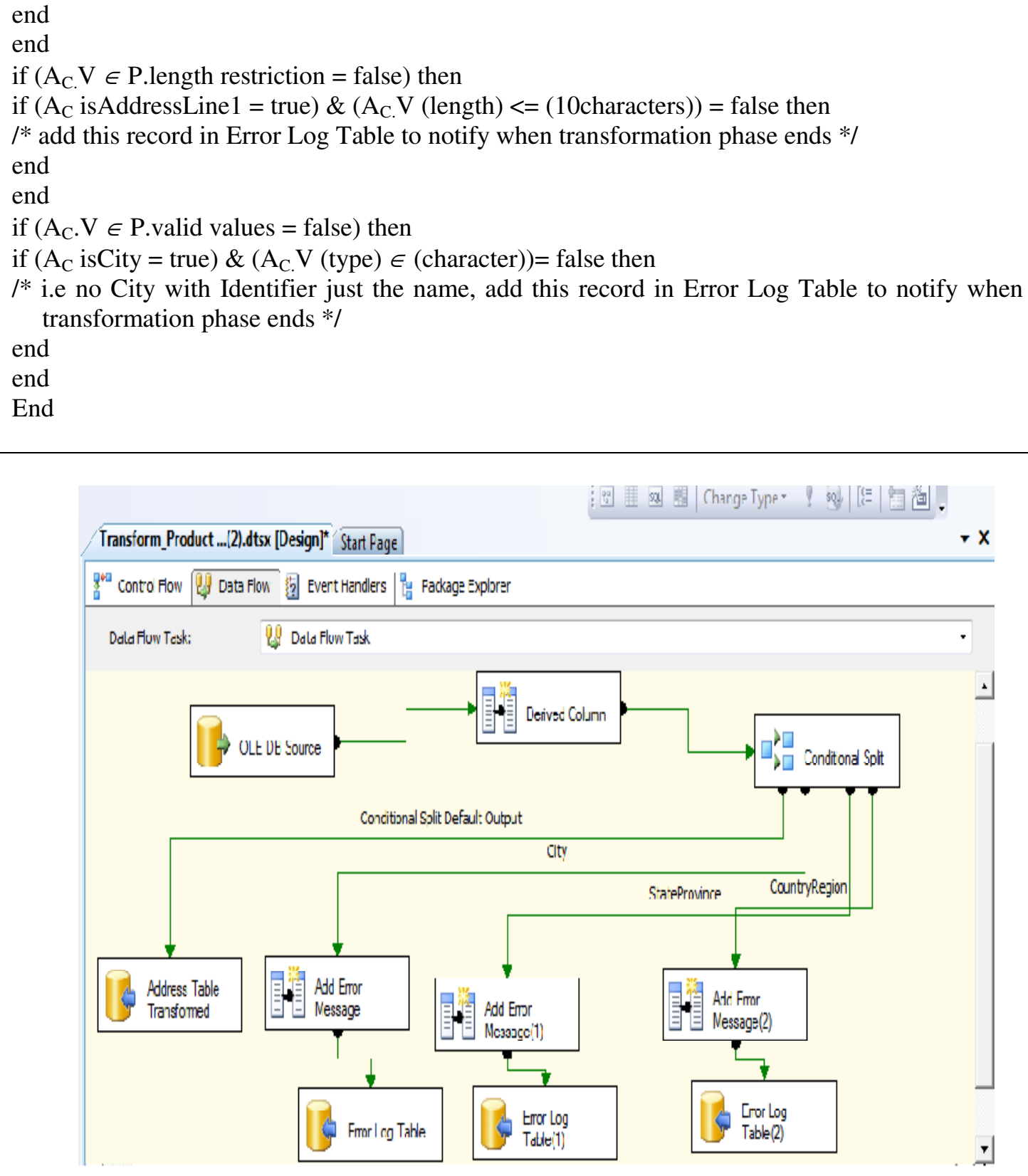

Figure 3. Deeper view for Address Table Transformation

If we compare the number of candidate keys before (Table 2) and after (Table 3), we will notice that previous gains from extract phase are still existing as well as additional improvement in the number of distinct values for our candidate keys resulting in better representation of the data to be finally loaded into the DW. 
International Journal of Data Mining \& Knowledge Management Process (IJDKP) Vol.3, No.6, November 2013

\begin{tabular}{|c|c|c|}
\hline Address Table & \multicolumn{2}{|c|}{ After Transforming } \\
\hline \multirow{4}{*}{$\begin{array}{l}\text { 1- Number of Candidate Keys and } \\
\text { Confidence }\end{array}$} & Key Columns & Key Strength \\
\hline & Address:D & $100 \%$ \\
\hline & AddressLine1 & $100 \%$ \\
\hline & PostalCode & $99.9610 \%$ \\
\hline 2. Column Null Ratio & \multicolumn{2}{|c|}{$\cdot$} \\
\hline \multirow[t]{3}{*}{ 3- Top Column Value Distribution } & Address $\mid R$ & 100062 \\
\hline & AddressLine1 & 100062 \\
\hline & PostalCodes & 100023 \\
\hline 4- Functional dependency & \multicolumn{2}{|c|}{$\begin{array}{l}\text { There is still } 2 \text { columns with full functional } \\
\text { dependency capabilities "Address|Dand } \\
\text { AddressLine1" }\end{array}$} \\
\hline
\end{tabular}

Table 3. Behavior of Address Table after Transformation

\section{SCHEDULing ALGORITHMS}

Following the approaches proposed to optimize the ETL process, and more specifically the "load" phase of this stage, we decided to focus on 3 scheduling techniques where each represents a different perspective of data processing. They are "First-in-First-Out"(FIFO), Minimum Cost (MC), and Round Robin (RR). We will first introduce each one of them and then explain how they were mapped on our data. Those techniques were used at different stages and in the following section we will show the what-if scenarios results on our test data.

\subsection{FIRST IN FIRST OUT}

First In First Out (FIFO) is one of the very primitive algorithms that simply takes the data as soon as it comes and transfers it to the destination regardless of any priorities. The input to the algorithm is simply all tables required for the DW, and the output is their successful transfer. Our implemented algorithm proceeds as follow: First, all queries of those tables (Tnq) are added to osne list (AL.FIFO.Tnq) where each query represents the selection of all columns of the table(T), then all tables names (Tn) are added to the same list. For each query in the list "AL.FIFO.Tnq" a connection to the Database holding the table was created and then we started measuring the difference between the start time (S.T) and end time (E.T) for processing the query "Table Total Execution Time" $\left(\mathrm{E}_{\mathrm{T}}\right)$. At the end we added all those $\mathrm{E}_{\mathrm{T}}$ together to have the total time $\mathrm{T}_{\text {tot }}$ to load all data using FIFO technique over different stages.

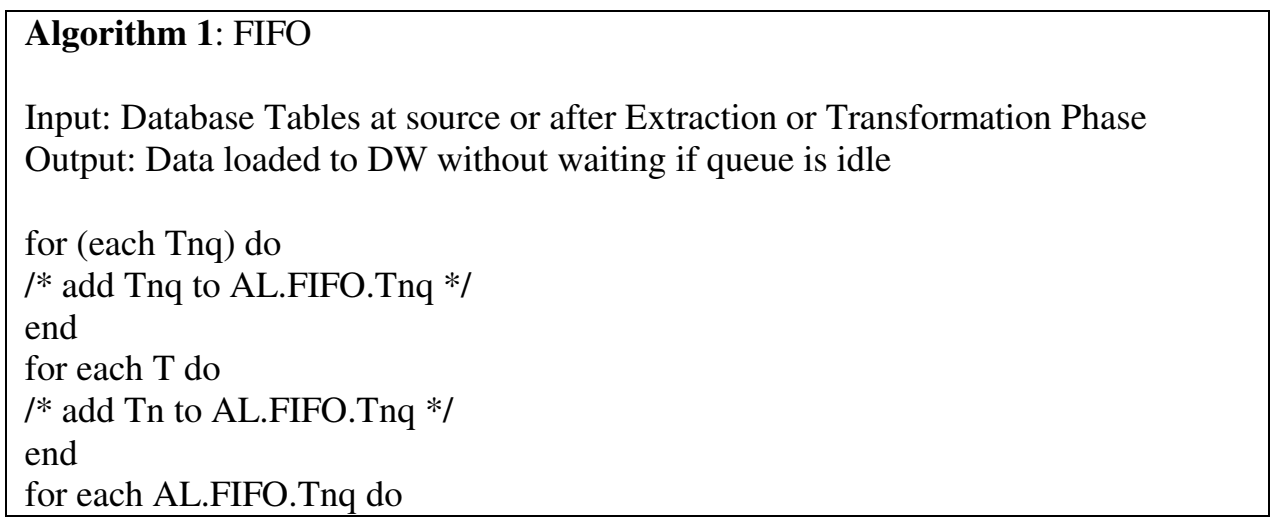




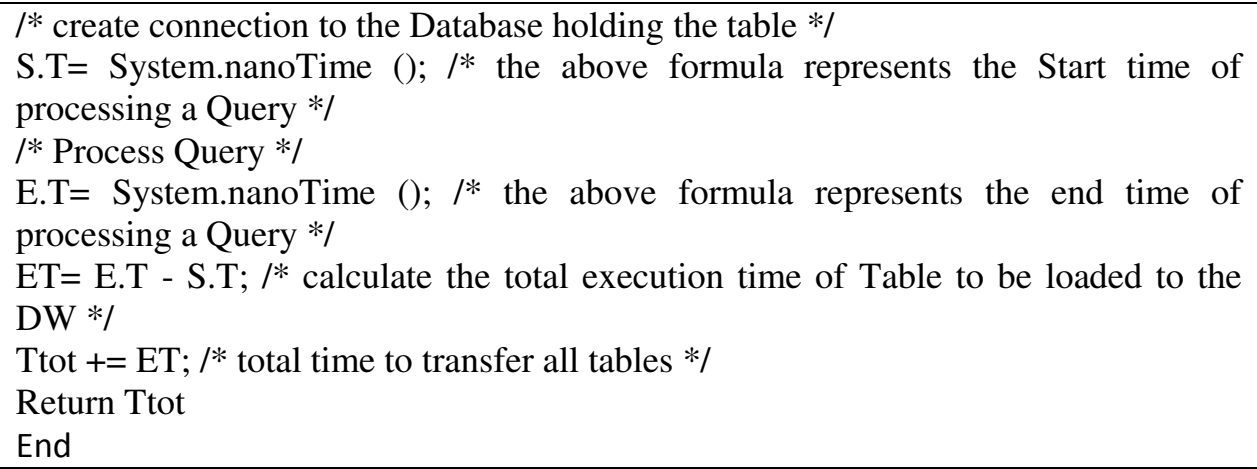

\subsection{MINIMUM COST}

The Minimum Cost (MC) scheduling is the second proposed algorithm to reduce the time needed for the execution of the loading phase. Similar to the FIFO algorithm, MC takes as input the data from any stage of Extract/Transform or at sources and as output the successful transfer of data but based on those with maximum volume first. Initially, after we specify the list of Tables (T) required we add all their names (Tn) to a list (AL.Tn). Afterwards, we process each table to retrieve its size (Ts) to add it beside (Tn) and its query (Tnq) to one list (AL.MC.Tnqs). Then, we take this list and re-sort it in a descending order (AL.MC.Tnqs.Desc) based on the size. Finally, once the list is ready, we create another connection to each table in this list and start measuring the difference between its start time (S.T) and its end time (E.T) to get our total table execution time $\mathrm{E}_{\mathrm{T}}$ and their summation leads us to the total time $\mathrm{T}_{\text {tot }}$ needed for $\mathrm{MC}$ algorithm to finish its job.

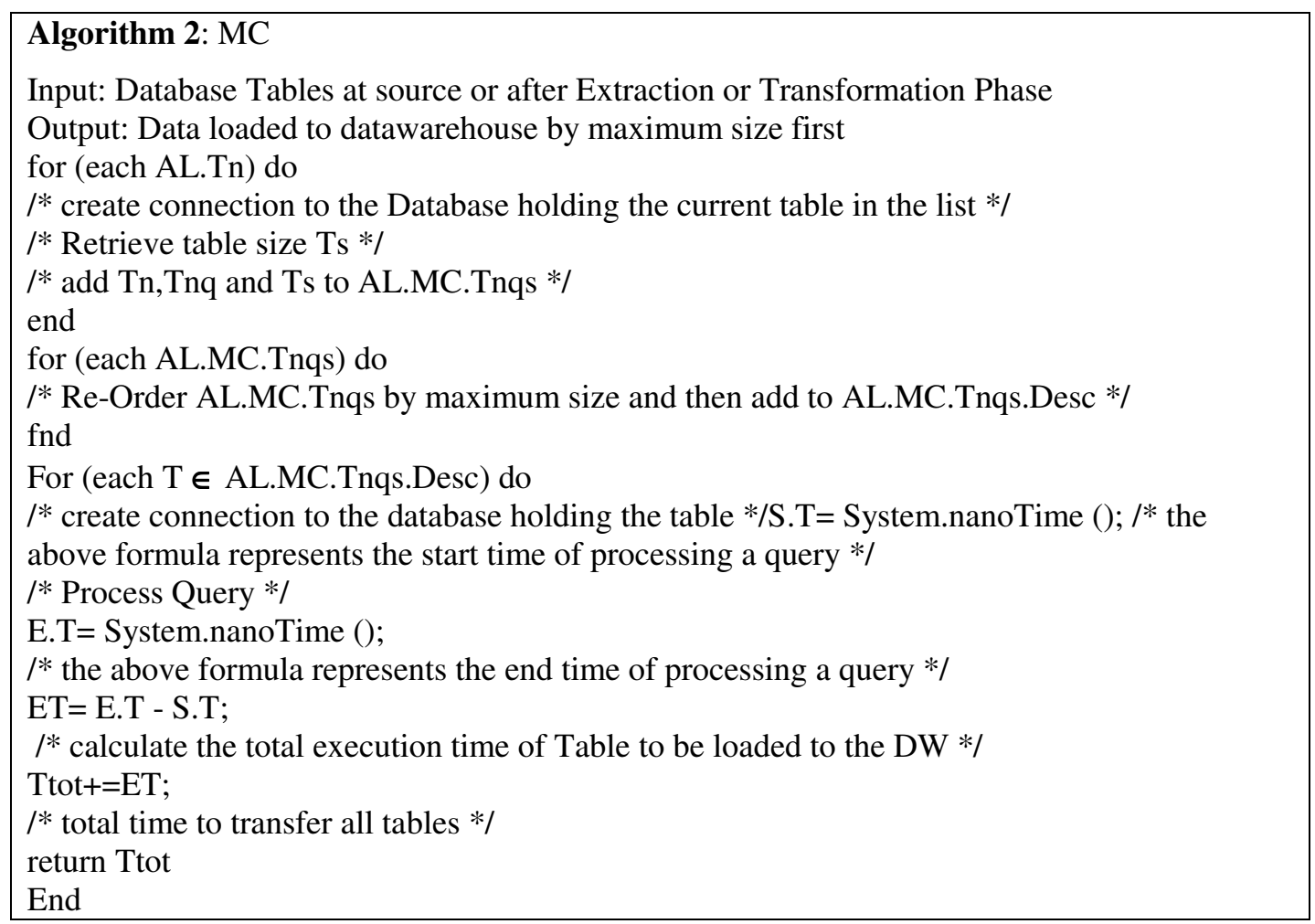




\subsection{ROUND ROBIN}

Our third technique is Round Robin (RR) which we implemented in two version rather than the traditional one to analyze their behaviors. So, instead of implementing the traditional Round Robin based on assigning time slices in equal portions for every table. We also implemented another version based on fixed threshold number of records to get a new perspective about what if having to wait for processing a complete set of records regardless of their size as the rotation factor.

\subsubsection{TIME BASED ROUND ROBIN (TRR)}

In the first version of RR we started with setting rotations based on time, thus as the pervious algorithms the input is the data from any stage of Extract/Transform or at sources along with the time slice. The algorithm starts by creating connections to all tables to be loaded and at the same time setting their status initially to false (i.e. idle status) until they get processed. Thus, when the table status (S) changes to True we will set the current time (C.T) value to be the start time (S.T) of the table.Then we check if the table was fully processed or not by comparing an incremental count of table records (C.Tr) with its total size (Ts). If there is still unprocessed records we check if this table was partially processed before to avoid miss-capturing of table actual start time by verifying the status of the indicator (ind) assigned to this table which initially is set to 0 (i.e. table was never processed). Afterwards, as long as rotation turn isn't reached (C.T is less than sum of S.T and TRR) and the table is not fully processed (C.Tr is not equal to Ts), we will process the records using the table query (Tnq) while adjusting table C.T value. Once the table gets fully processed we capture the table end time (E.T) and calculate the difference to get the total table execution time $\left(\mathrm{E}_{\mathrm{T}}\right)$.At the end we add all those $\left(\mathrm{E}_{\mathrm{T}}\right)$ together to have the total time $\mathrm{T}_{\text {tot }}$ to load the tables.

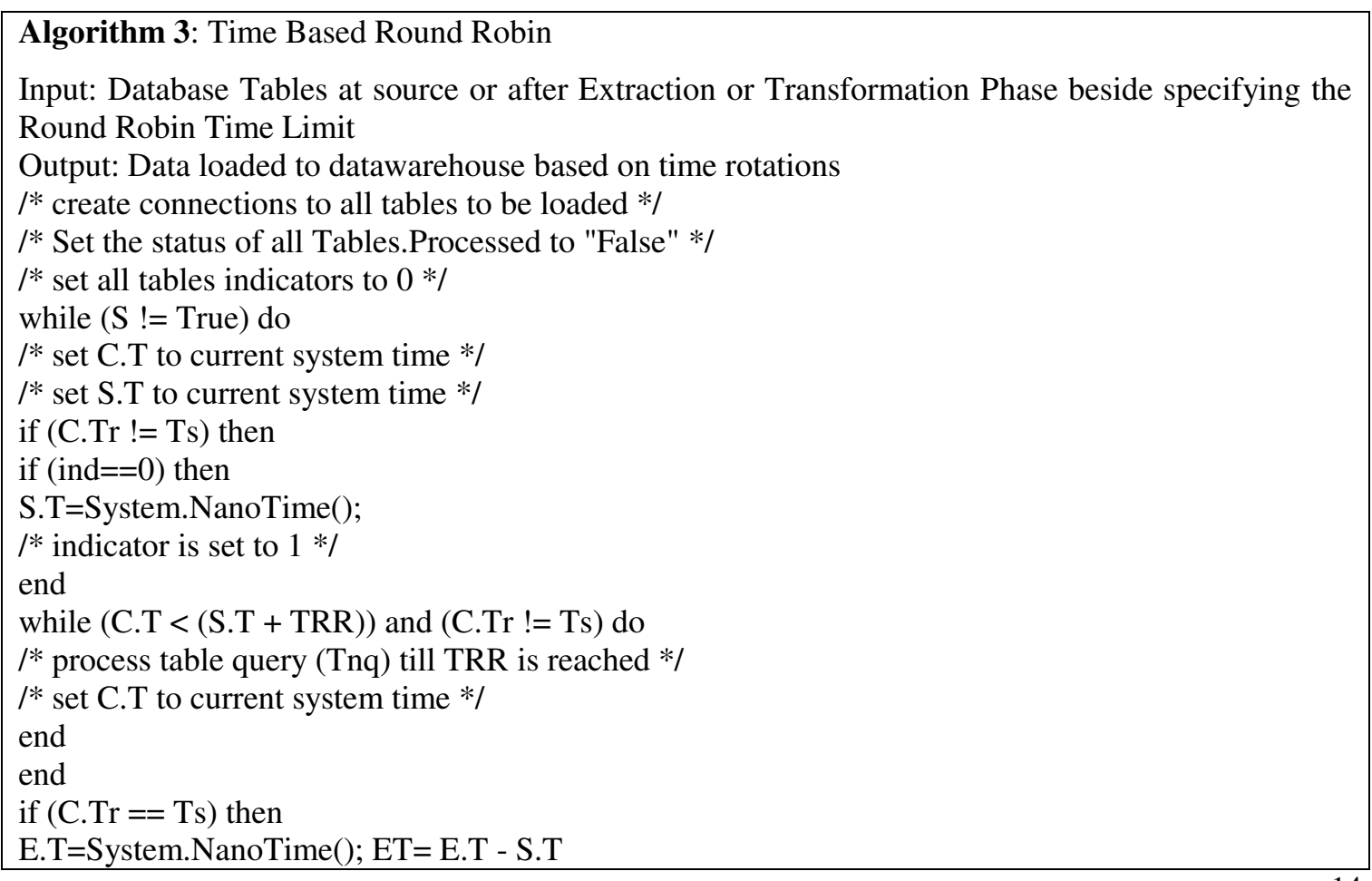




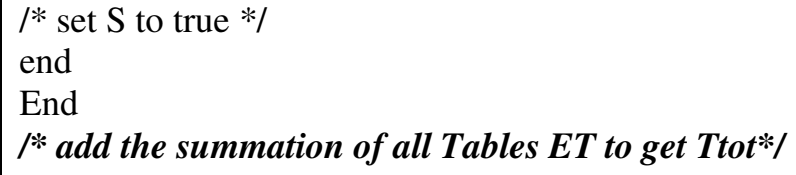

\subsubsection{RECORD LIMIT BASED ROUND ROBIN}

So as with prior techniques we take as input the data coming from any stage of Extract/Transform or at sources along with the Round Robin records limit (LRR) for rotation. First, we create a connection to all the tables to be transferred then as long as we didn't finish processing all the data we set our Round Robin status (S) to false then we start capturing the start time (S.T) of processing a table and change its status to true (T). While the Round Robin Limit (LRR) is not reached and we haven't finished processing the whole table size (Ts), the query referring to all table's data (Tnq) get executed. Then, when we finish loading all the table we capture its end time (E.T) then calculate the table total execution time $\left(\mathrm{E}_{\mathrm{T}}\right)$ and add it to a list of all tables total execution time $\left(\mathrm{Al} . \mathrm{E}_{\mathrm{T}}\right)$. Finally when all tables are loaded we adjust our algorithm end round robin status $(\mathrm{S})$ to "True" and from $\left(\mathrm{AL} . \mathrm{E}_{\mathrm{T}}\right)$ we get our Total Time $\left(\mathrm{T}_{\mathrm{tot}}\right)$ of Round Robin based on records limit technique.

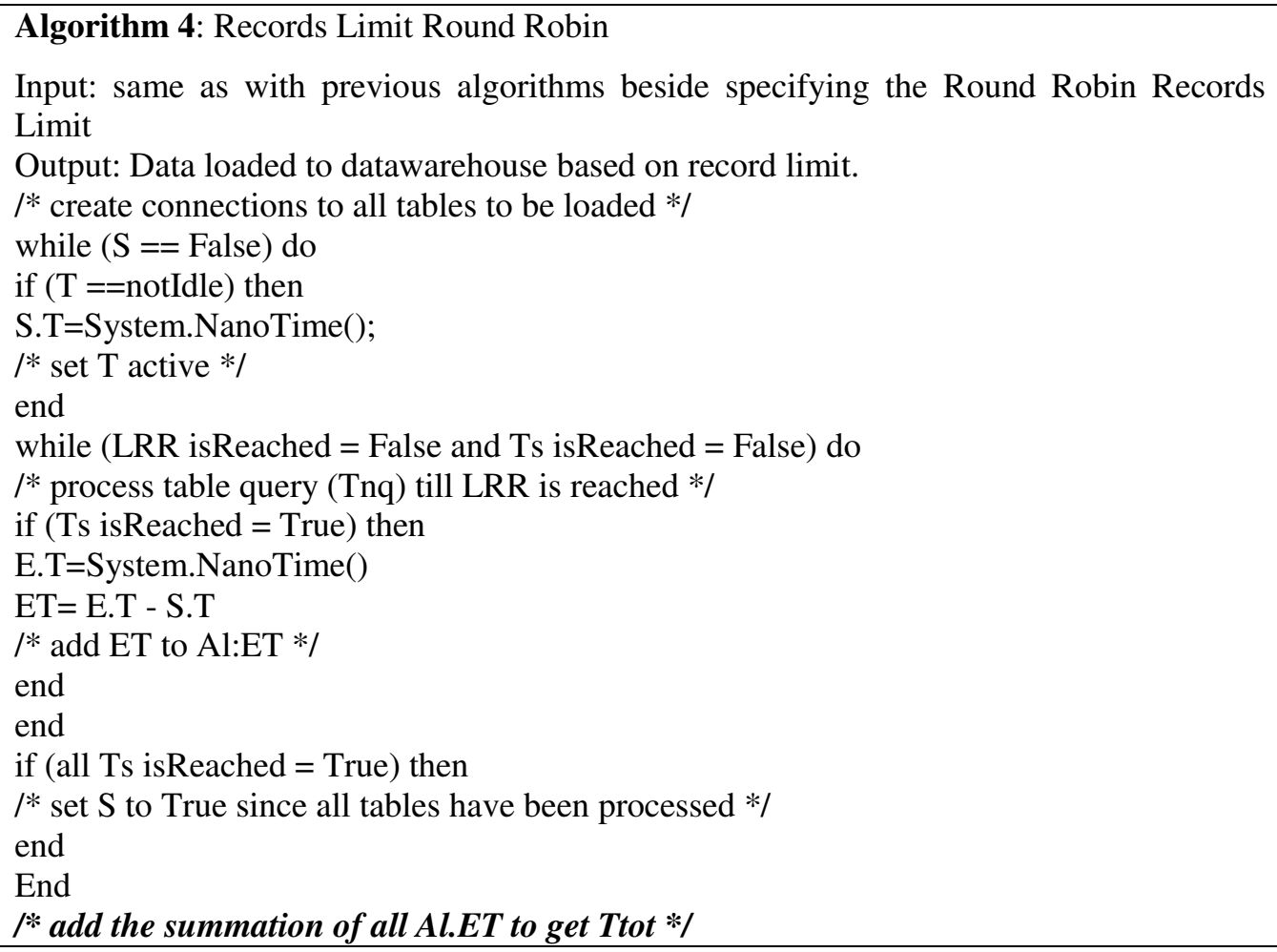

\section{SCHEDULING EXPERIMENTS}

In this section, we discuss the experimental results of our proposed algorithms. The used data was from AdventureWorks Database [http://msftdbprodsamples.codeplex.com/] to simulate the loading phase to a sales DW. Our objective is to evaluate data transfer using different techniques 
(FIFO, MC, RR time and record rotation). The data included in our test is coming from data at their sources after extraction and transform phases as we wanted to capture the time needed to transfer data from each stage and which technique is the most suitable in case there is a decision required. For choosing FIFO, FIFO turns to be a typical solution if we went random with just a simple knowledge about the data in hand which sometime might be the case with the need for fast response for critical inquiries. As for MC, this one targets large data sets first which requires having sufficient memory allocation. For the Round Robin, we tried to look not only at the traditional time rotation but also what if we used specified number of records as our limit.

All experiments have been conducted on a Core i7 with $2.5 \mathrm{GHz}$ and $16 \mathrm{~GB}$ main memory. As for the Database size over different stages: $14 \mathrm{~GB}$ for data at sources, 6.3 GB at extract and 6.89 GB at Transform. From those experiments, we noticed as shown in figure 4.a and 4.b that when comparing all scheduling techniques that FIFO has slightly better performance than MC followed by Time Based Round Robin, while Record Limit Based Round Robin behaves the worst. However, when increasing the record limit as shown in figure 5.a and figure 5.b, the performance improves which can be taken into consideration for scenarios where there is a need to quickly load part of the data set into a data mart. On the other hand, after testing several Time Based Round Robin as shown in figure 6.a and 6.b, we concluded that it behaves best with smaller data set (as with Extracted Data Set).

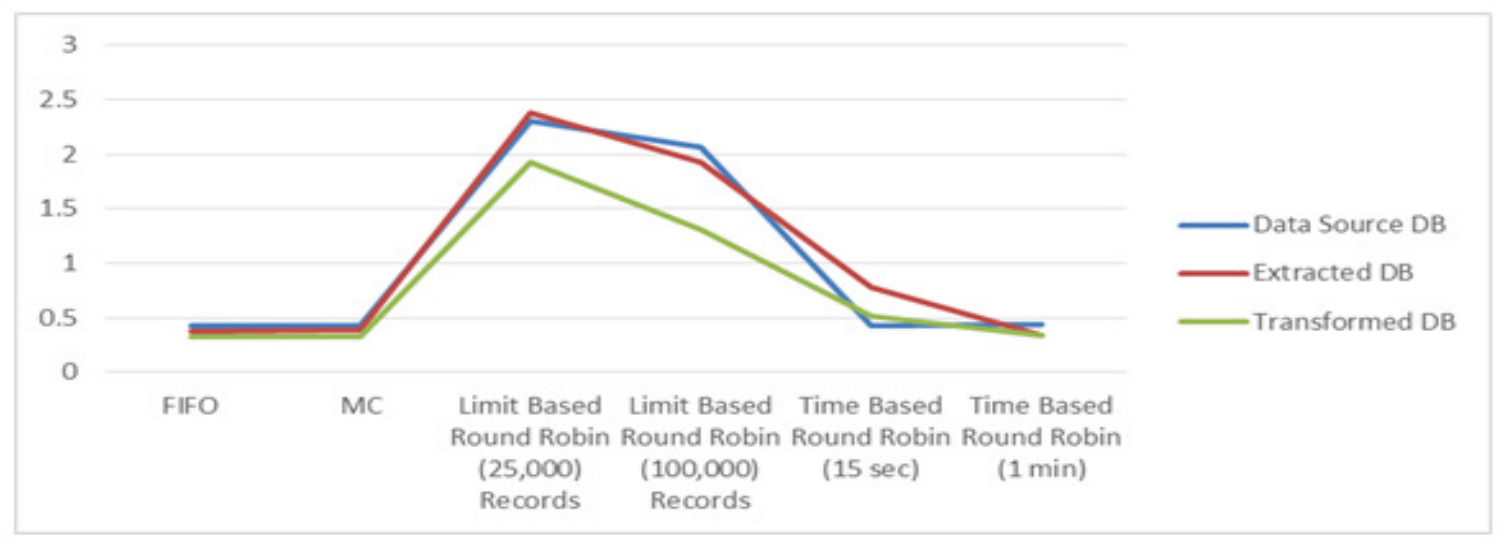

Figure 4.a Scheduling Techniques by Minutes for Data Loaded at Different Stages

\begin{tabular}{|l|l|l|l|}
\hline FIFO & 0.427844281 & 0.374048844 & 0.324229626 \\
\hline MC & 0.432603715 & 0.388010876 & 0.329426132 \\
\hline Limit Based Round Robin $(25,000)$ Records & 2.306548228 & 2.386927216 & 1.927858971 \\
\hline Limit Based Round Robin $(50,000)$ Records & 2.490471779 & 2.333232646 & 1.794040655 \\
\hline Limit Based Round Robin $(75,000)$ Records & 2.398116768 & 2.238604187 & 1.682960375 \\
\hline Limit Based Round Robin $(100,000)$ Records & 2.071130743 & 1.922173568 & 1.307447341 \\
\hline Time Based Round Robin $(15 \mathrm{sec})$ & 0.423583402 & 0.77385139 & 0.512963346 \\
\hline Time Based Round Robin $(30 \mathrm{sec})$ & 0.432563809 & 0.332146974 & 0.369322797 \\
\hline Time Based Round Robin $(45 \mathrm{sec})$ & 0.421170103 & 0.382187158 & 0.338832748 \\
\hline Time Based Round Robin $(1 \mathrm{~min})$ & 0.435068669 & 0.33775838 & 0.333256225 \\
\hline
\end{tabular}

Figure 4.b Scheduling Techniques Statistics by Minutes for Data Loaded at Different Stages 
International Journal of Data Mining \& Knowledge Management Process (IJDKP) Vol.3, No.6, November 2013

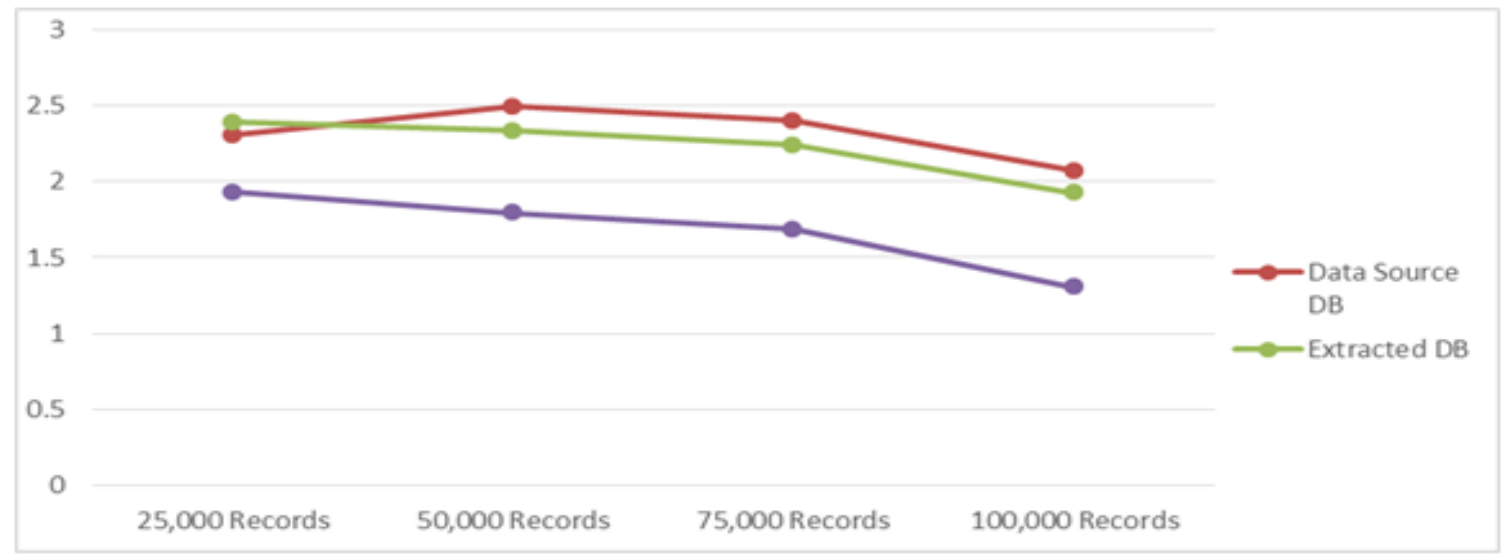

Figure 5.a Records Limit Based Round Robin for Data Loaded at Different Stages

\begin{tabular}{|l|l|l|l|}
\hline FIFO & 0.427844281 & 0.374048844 & 0.324229626 \\
\hline MC & 0.432603715 & 0.388010876 & 0.319426132 \\
\hline Limit Based Round Robin (25,000) Records & 2.306548228 & 2.386927216 & 1.927858971 \\
\hline Limit Based Round Robin (50,000) Records & 2.490471779 & 2.333232646 & 1.794040655 \\
\hline Limit Based Round Robin (75,000) Records & 2.398116768 & 2.238604187 & 1.682960375 \\
\hline Limit Based Round Robin (100,000) Records & 2.071130743 & 1.922173568 & 1.307447341 \\
\hline Time Based Round Robin (15 sec) & 0.423583402 & 0.77385139 & 0.512963346 \\
\hline Time Based Round Robin (30 sec) & 0.432563809 & 0.332146974 & 0.369322797 \\
\hline Time Based Round Robin (45 sec) & 0.421170103 & 0.382187158 & 0.338832748 \\
\hline Time Based Round Robin (1 min) & 0.435068669 & 0.33775838 & 0.333256225 \\
\hline
\end{tabular}

Figure 5.b Records Limit Based Round Robin Statistics for Data Loaded at Different Stages

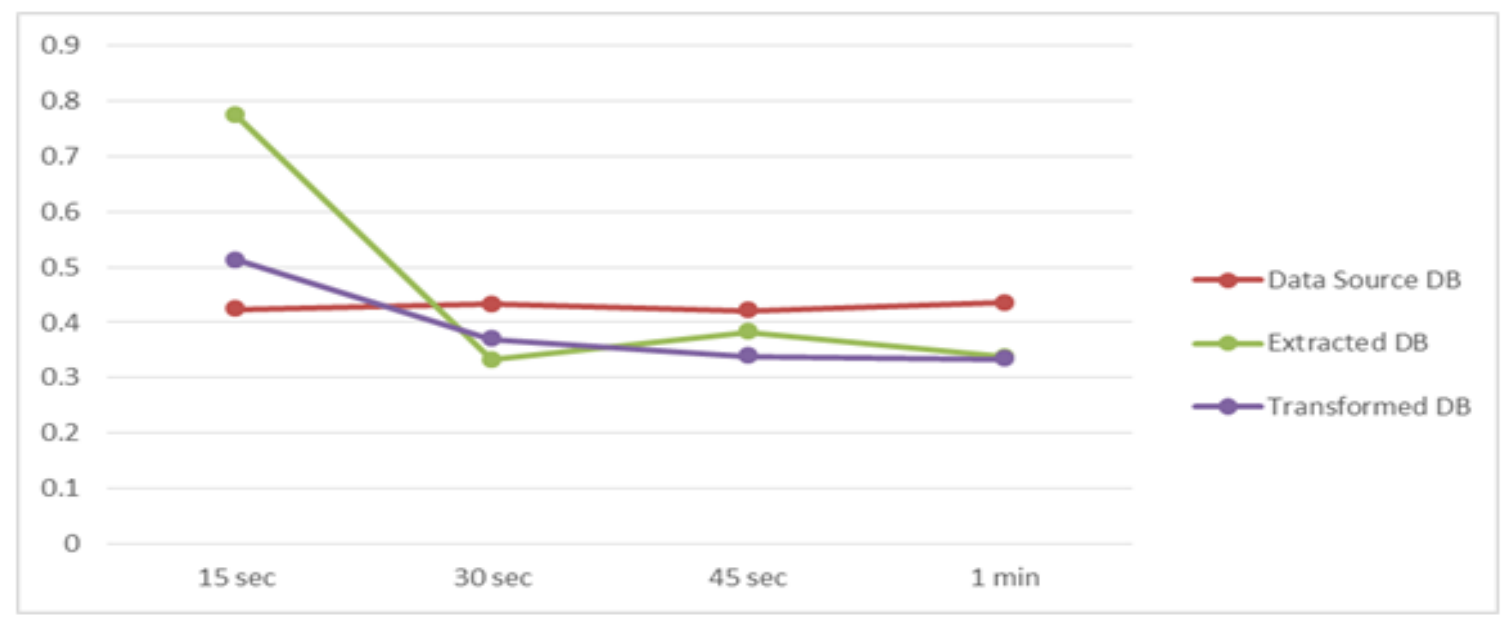

Figure 6.a Time Based Round Robin for Data Loaded at Different Stages 


\begin{tabular}{|l|l|l|l|}
\hline Time Based Round Robin & Data Source DB & Extracted DB & $\begin{array}{c}\text { Transformed } \\
\text { DB }\end{array}$ \\
\hline $15 \mathrm{sec}$ & 0.423583402 & 0.77385139 & 0.512963346 \\
\hline $30 \mathrm{sec}$ & 0.432563809 & 0.332146974 & 0.369322797 \\
\hline $45 \mathrm{sec}$ & 0.421170103 & 0.382187158 & 0.338832748 \\
\hline $1 \mathrm{~min}$ & 0.435068669 & 0.33775838 & 0.333256225 \\
\hline
\end{tabular}

Figure 6.b Time Based Round Robin Statistics for Data Loaded at Different Stages

\section{CONCLUSION AND FUTURE WORK}

In a typical DW environment, data is extracted periodically from the applications that support business processes and copied to special dedicated machines. There it can be validated, reformatted, reorganized, summarized, restructured, and supplemented with data from other sources which will lead to having a DW acting as the main source of information for future analysis, report generation, and presentation through ad-hoc reports, portals, and dashboards. In this paper, we introduced a new approach to enhance performance using semantics for Extraction and Transformation which reside in the staging area just before the final loading phase. We proposed a semantics-based algorithm for each phase and presented the statistical results of applying those algorithms on the "Sales" schema. The data profiling tool results show that incorporating semantics in the staging area has an obvious impact on the quality of the resulting data that is presented to the loading phase. We also think that the ontologies presented in this work are tailored for the sales case study, however, different businesses imply different ontologies that need to be considered.As for loading data continuous attempts to select the best and most convenient approach to data transfer will vary depending on the data in hand as well as available resources such as CPU and main memory beside the urgency factor. In this work we tried to analyze and evaluate different scheduling techniques namely, FIFO (Random), MC (Maximum Size First), RR (based on time), and finally a new approach for RR which that is based on rotating on fixed number of records regardless of their size. For future work, we would like to consider Minimum Memory (MM) and other algorithms. In addition implementing those algorithms in a distributed environment is also a possible direction for future work.

\section{REFERENCES}

[1] El-Gamal, N.: Data warehouse conceptual modeling approaches. In: Proceedings of the 37th International Conference on Computers and Industrial Engineering, Alexandria, Egypt (October 2007) 231-242

[2] Inmon, W.H.: Building the data warehouse. Wiley Publishing,Inc., Wellesley, MA,USA (1992)

[3] Server, M.S.: Data profiling task and viewer. http://technet.microsoft.com/en-us/library/bb895310.aspx (2013)

[4] Knight, B., Veerman, E., Dickinson, G., Hinson, D., Herbold, D.: Professional Microsoft SQL Server 2008 Integration Services. Wiley Publishing, Inc. (2008)

[5] Bateni, Mohammad Hossein and Golab, Lukasz and Hajiaghayi, Mohammad Taghi and Karloff, Howard. Scheduling to Minimize Staleness and Stretch in Real-time Data Warehouses. In Proceedings of the twenty-first Annual Symposium on Parallelism in Algorithms and Architectures, 2009. 
International Journal of Data Mining \& Knowledge Management Process (IJDKP) Vol.3, No.6, November 2013

[6] Furtado P. Experimental Evidence on Partitioning in Parallel Data Warehouses. In Proceedings of the 7th ACM International Workshop on Data Warehousing and Olap, 2004.

[7] Simitsis, A., Vassiliadis, P., Sellis, T.: Optimizing etl processes in data warehouses.In: Data Engineering, 2005. ICDE 2005. Proceedings. 21st International Conference on. (2005) 564-575.

[8] Ladjel, B., Michel, S., Herv, L., Mukesh, M.: Bringing together partitioning, materialized views and indexes to optimize performance of relational data warehouses.In Kambayashi, Y., Mohania, M., W, W., eds.: Data Warehousing and Knowledge Discovery. Volume 3181 of Lecture Notes in Computer Science. Springer Berlin Heidelberg (2004) 15-25.

[9] Thi, A.D.H., Nguyen, B.T.: A semantic approach towards cwm-based etl processes.In: Proceedings of I-SEMANTICS 08, Graz, Austria (September 2008) 58-66.

[10] Skoutas, D., Simitsis, A., Sellis, T.: Journal on data semantics xiii. Springer-Verlag, Berlin, Heidelberg (2009) 120-146.

[11] Skoutas, D., Simitsis, A.: Ontology-based conceptual design of etl processes for both structured and semi-structured data. International Journal on Semantic Web and Information Systems (IJSWIS) 3(4) (2007) 1-24.

[12] Santos, Ricardo Jorge and Bernardino, Jorge. Optimizing Data Warehouse Loading Procedures for Enabling Useful-time Data Warehousing. In Proceedings of the 2009 International Database Engineering and Applications Symposium, 2009.

[13] Thiele, Maik and Bader, Andreas and Lehner, Wolfgang. Multi-Objective Scheduling for Real-Time Data Warehouses. In Business, Technologie Und Web (BTW), 2009.

[14] Thomas Jorg and Stefan Dessloch. Formalizing ETL Jobs for Incremental Loading of Data Warehouses. In Datenbanksysteme in Business, Technologie Und Web (BTW), 13.Münster, Germany, 2009.

[15] Fenk, Robert and Kawakami, Akihiko and Markl, Volker and Bayer, Rudolf and Osaki, Shunji. Bulk Loading a Data Warehouse Built Upon a UB-Tree. In Proceedings of the 2000 International Symposium on Database Engineering and Applications, 2000.

[16] Mallikharjuna Reddy V and Sanjay K. Jena. Active Datawarehouse Loading by Tool Based ETL Procedure. In Proceedings of the 2010 International Conference on Information and Knowledge Engineering, july 12-15, Las Vegas Nevada, USA, 2010.

[17] Costa, Marco and Madeira, Henrique. Handling Big Dimensions in Distributed Data Warehouses Using the DWS Technique. In Proceedings of the 7th ACM International Workshop on Data Warehousing and Olap, 2004.

[18] Anastasios Karagiannis, Panos Vassiliadis, Alkis Simitsis. Macro Level Scheduling of ETL Workflows. In 9th International Workshop on Quality in Databases (QDB 2011), in Conjunction with VLDB, 2011. 